\title{
Progresso genético com a seleção recorrente recíproca para híbridos interpopulacionais de milho
}

\author{
Matheus Costa dos Reis ${ }^{(1)}$, João Cândido de Souza(1), Magno Antônio Patto Ramalho(1), \\ Fernando Lisboa Guedes ${ }^{(1)}$ e Pedro Henrique Araújo Diniz Santos ${ }^{(1)}$
}

\begin{abstract}
(1)Universidade Federal de Lavras, Departamento de Biologia, Caixa Postal 3037, CEP 37200-000 Lavras, MG. E-mail: matheuscreis@yahoo.com.br, cansouza@dbi.ufla.br, mapramalho@dbi.ufla.br, fbrguedes@yahoo.com.br e phsantos2004@yahoo.com.br
\end{abstract}

\begin{abstract}
Resumo - O objetivo deste trabalho foi avaliar o progresso genético na obtenção de híbridos interpopulacionais com a seleção recorrente recíproca. Na safra 2005/2006, foram avaliados os híbridos interpopulacionais de milho (Zea mays) originados de três ciclos de seleção $(0,1$ e 2$)$ iniciados em 2003, os híbridos simples parentais e o híbrido duplo de F1, em blocos ao acaso com cinco repetições, em dois locais. Na safra seguinte, somente os híbridos interpopulacionais dos ciclos 0 e 2 foram avaliados nas mesmas condições, com 40 repetições por local. O híbrido interpopulacional apresentou desempenho equivalente ao do melhor híbrido parental simples em poucos ciclos seletivos. As estimativas do progresso genético por ciclo foram de 7,9\% (ou 0,7 Mg ha-1) para produtividade de espigas despalhadas e de 3,5\% para prolificidade. É possível inferir que a seleção recorrente recíproca é eficiente em elevar a produção de híbridos interpopulacionais obtidos a partir de populações $F_{2}$ de híbridos simples de milho.
\end{abstract}

Termos para indexação: Zea mays, agricultura familiar, híbrido simples, resposta à seleção.

\section{Genetic progress with reciprocal recurrent selection for interpopulation hybrids of maize}

\begin{abstract}
The objective of this work was to evaluate the genetic progress obtained in interpopulation hybrids with the reciprocal recurrent selection. During the 2005/2006 planting season, maize (Zea mays) interpopulation crosses from three selection cycles $(0,1$ and 2$)$ initiated in 2003, single-cross parental hybrids and the double-cross $F_{1}$ hybrid were evaluated in randomized blocks with five replicates, at two locations. In the next planting season, only interpopulational hybrids from cycles 0 and 2 were evaluated under the same conditions, with 40 replicates per location. The interpopulation hybrid had a yield performance equivalent to that of the best single-cross parent in few selection cycles. The estimates of genetic progress per cycle were of $7.9 \%$ ( or $0.7 \mathrm{Mg} \mathrm{ha}^{-1}$ ) for unhusked ear yield and of $3.5 \%$ for prolificacy. It is possible to infer that the reciprocal recurrent selection effectively improves the yield of interpopulational hybrids obtained from $\mathrm{F}_{2}$ generations of single-cross maize hybrids.
\end{abstract}

Index terms: Zea mays, family agriculture, single-cross hybrids, response to selection.

\section{Introdução}

A cultura do milho no Brasil possui enorme diversidade de manejo. Em uma mesma região, convivem agricultores de alta tecnologia, que utilizam todos os insumos disponíveis, e os de agricultura familiar, que utilizam poucos insumos. Essa diferença de manejo é visível, principalmente com relação à semente utilizada. Os empresários utilizam os melhores híbridos comerciais, enquanto os agricultores familiares utilizam cultivares sem o potencial produtivo desses híbridos ou até mesmo os grãos colhidos na safra anterior como semente. Para que os agricultores familiares possam ter maior lucro, é indispensável que aproveitem a heterose na sua integralidade.

Para isso, é necessário que a semente híbrida seja acessível, sobretudo no que se refere ao custo. Uma opção são os híbridos interpopulacionais, como demonstrado por Souza Sobrinho et al. (2002). Esses híbridos podem ser obtidos de populações derivadas de híbridos simples comerciais que apresentem grande vigor quando cruzadas. Adicionalmente, pode-se promover a seleção recorrente recíproca (SRR) entre essas populações, para aumentar a capacidade de combinação entre elas. 
A SRR com uso de populações derivadas de híbridos simples já se mostrou viável em outras condições (Coors, 1999; Raposo et al., 2004). No entanto, é necessário utilizar um método de SRR que seja mais dinâmico e rápido. Foram propostas várias alternativas de condução da SRR além da original de Comstock et al. (1949). A maioria dessas alternativas foi desenvolvida no Brasil (Paterniani \& Vencovsky, 1977, 1978; Souza Júnior, 1987). A eficiência do emprego desses métodos de condução da SRR ainda não foi avaliada, sobretudo quando se utilizam populações derivadas de híbridos simples comerciais.

O objetivo deste trabalho foi avaliar o progresso genético entre populações derivadas de híbridos simples com a SRR para o incremento de produtividade de grãos do híbrido interpopulacional.

\section{Material e Métodos}

Oprograma de SRR foirealizado na área experimental da Universidade Federal de Lavras, nas coordenadas $21^{\circ} 14^{\prime} \mathrm{S}$ e $40^{\circ} 17^{\prime} \mathrm{W}$, a $918 \mathrm{~m}$ de altitude. As duas populações utilizadas neste estudo, denominadas 1 e 2, são derivadas de dois híbridos simples (HS) comerciais e foram obtidas pelo intercruzamento ao acaso de 3.000 plantas $F_{1}$ de cada HS em dois campos isolados, que gerou duas populações $F_{2}$ em equilíbrio de Hardy-Weinberg (Wricke \& Weber, 1986).

O método empregado no programa de SRR foi semelhante ao proposto por Souza Júnior (1987). Para isso, iniciou-se o primeiro ciclo seletivo (ciclo $0-\mathrm{C} 0$ ) semeando, em março de 2003, duas mil sementes de cada população, em dois blocos contínuos. As plantas prolíficas de ambas as populações tiveram suas espigas inferiores autofecundadas, e as superiores foram cruzadas aos pares, com plantas da população recíproca, o que gerou uma progênie $S_{1}$ e uma progênie de irmãos germanos interpopulacionais em cada genótipo (planta). As sementes das progênies $S_{1}$ foram reservadas para compor a unidade de recombinação, $\mathrm{e}$ as progênies de irmãos germanos tiveram parte de suas sementes utilizada para avaliação e parte estocada para compor o híbrido interpopulacional do $\mathrm{C} 0\left(\mathrm{CO}_{1} \times \mathrm{CO}_{2}\right)$.

$\mathrm{Na}$ safra 2003/2004, 121 progênies de irmãos germanos interpopulacionais foram avaliadas em látice simples $11 \times 11$. As parcelas eram constituídas de uma linha de $3 \mathrm{~m}$, espaçadas entre si em $0,8 \mathrm{~m}$, com densidade de semeadura de cinco plantas por metro após o desbaste. Selecionaram-se 10\% das progênies, tendo-se considerado principalmente a produtividade média (peso de espigas despalhadas). Para possibilitar a conclusão de um ciclo por ano, foi necessário semear o campo para a realização da recombinação em março de 2004, antes que os dados utilizados na seleção fossem obtidos. Isso porque as baixas temperaturas na época do inverno não permitem o desenvolvimento adequado das plantas. Para isso, foi utilizada uma amostra de 100 sementes de cada uma das progênies intrapopulacionais $\mathrm{S}_{1}$ de ambas as populações. Em maio de 2004, já de posse dos dados e antes do florescimento das progênies, foram identificadas as progênies a serem recombinadas e as demais foram eliminadas. Simultaneamente foram obtidas as progênies do segundo ciclo (ciclo $1-\mathrm{C} 1$ ).

Diferentemente do ciclo anterior, no $\mathrm{C} 1$ foram geradas, em plantas prolíficas, progênies de meios-irmãos intrapopulacionais na espiga inferior e de meios-irmãos interpopulacionais na espiga superior, com uso de uma mistura de pólen da própria população e da população recíproca, respectivamente. Para garantir a aleatorização dos gametas selecionados, a cada coleta de pólen foi amostrado um mesmo número de pendões por progênie. Cem plantas de cada população, que se apresentaram eretas e com bom enchimento de grãos nas duas espigas, foram amostradas para a obtenção das progênies intra e interpopulacionais. A amostra equitativa de sementes de todas as progênies de meios-irmãos intrapopulacionais em cada população representa, respectivamente, as populações $\mathrm{Cl}_{1} \mathrm{e} \mathrm{Cl}_{2}$. De modo análogo, parte das sementes das progênies de meios-irmãos interpopulacionais foi misturada para a obtenção do híbrido interpopulacional do $\mathrm{C} 1\left(\mathrm{Cl}_{1} \times \mathrm{Cl}_{2}\right)$. Com a outra parte das sementes, procedeu-se à avaliação do C1 na safra 2004/2005, em experimentos conduzidos no delineamento látice 10x10 com três repetições, seguindo-se os mesmos procedimentos realizados na safra anterior. Foi considerada principalmente a produtividade de espigas despalhadas para a seleção das $15 \%$ melhores progênies.

Utilizando-se as sementes das progênies de meios-irmãos intrapopulacionais relacionadas com as progênies interpopulacionais selecionadas em cada população, iniciou-se o terceiro ciclo seletivo (Ciclo $2-\mathrm{C} 2$ ). O procedimento foi o 
mesmo realizado para a obtenção do $\mathrm{C} 0$, o que gerou novamente progênies $S_{1}$ e progênies de irmãos completos interpopulacionais, e foi conduzido na localidade de Ituiutaba, MG, onde as condições climáticas no período de inverno não interferem no desenvolvimento normal das plantas. Parte das sementes das progênies interpopulacionais foi utilizada para avaliação na safra de 2005/2006 do C2 e parte foi reservada para compor o híbrido interpopulacional do $\mathrm{C} 2\left(\mathrm{C} 2_{1} \times \mathrm{C} 22_{2}\right)$.

Para avaliar o progresso genético, foi retirada uma amostra equitativa de sementes de cada progênie interpopulacional avaliada por ciclo, que representou os híbridos interpopulacionais dos $\mathrm{C} 0, \mathrm{C} 1$ e $\mathrm{C} 2$ $\left(\mathrm{CO}_{1} \times \mathrm{CO}_{2}, \mathrm{Cl}_{1} \times \mathrm{Cl}_{2}\right.$ e $\left.\mathrm{C}_{1} \times \mathrm{C}_{2}\right)$. Adicionalmente, foram utilizados como testemunhas os HS parentais e o híbrido duplo $\left(\mathrm{HDF}_{1}\right)$ oriundo do cruzamento deles. Os seis tratamentos foram avaliados no delineamento em blocos ao acaso, com cinco repetições, em parcelas constituídas de duas linhas de $3 \mathrm{~m}$ espaçadas em $0,8 \mathrm{me}$ instaladas nos campos experimentais do Departamento de Biologia, e na Fazenda Experimental Vitorinha, da Universidade Federal de Lavras (Ufla), no município de Lavras, MG. A semeadura foi realizada na primeira quinzena do mês de novembro de 2005, no sistema de plantio direto, com $350 \mathrm{~kg} \mathrm{ha}^{-1}$ de fertilizantes na formulação 8-28-16 $\left(\mathrm{N}-\mathrm{P}_{2} \mathrm{O}_{5}-\mathrm{K}_{2} \mathrm{O}\right)$. Aos 21 dias após o plantio (DAP), foi realizado o desbaste das plantas, a fim de se obter um estande com 55.000 plantas $\mathrm{ha}^{-1}$, seguido da adubação de cobertura com $60 \mathrm{~kg} \mathrm{ha}^{-1} \mathrm{de} \mathrm{N}$. Os demais tratos culturais foram os recomendados para a cultura na região. As características avaliadas foram: produtividade de espigas despalhadas (PE), corrigida para $13 \%$ de umidade e para o estande ideal $\left(\mathrm{Mg} \mathrm{ha}^{-1}\right)$, e prolificidade (PLF), obtida pela razão entre o estande final e o número de espigas por parcelas.

O progresso genético foi avaliado em mais uma oportunidade, na safra de 2006/2007, quando apenas os híbridos interpopulacionais $\mathrm{C}_{1} \times \mathrm{C}_{2}$ e $\mathrm{C} 2_{1} \times \mathrm{C}_{2}$ foram avaliados no delineamento de blocos ao acaso, com 40 repetições e em parcelas de duas linhas de $4 \mathrm{~m}$ espaçadas em $0,6 \mathrm{~m}$. O plantio foi realizado na primeira quinzena de novembro de 2006, no sistema de plantio direto, e os tratos culturais, as características avaliadas e os locais de avaliação foram os mesmos utilizados nas avaliações da safra de 2005/2006.

Os dados foram submetidos à análise de variância, a 5\% de significância pelo teste $\mathrm{F}$ com auxílio do SAS versão 8.0 (SAS Institute, 2000), tendo-se considerado os efeitos de tratamentos e locais como fixos no modelo para as análises individuais de safras. Nas análises conjuntas de safras, foram utilizados somente os dados dos tratamentos $\mathrm{CO}_{1} \times \mathrm{CO}_{2}$ e $\mathrm{C} 21_{1} \times \mathrm{C} 2{ }_{2}$. O progresso genético por ciclo de seleção foi estimado de dois modos: i, por meio da equação de regressão, com os dados da safra de 2005/2006, em que o desempenho do híbrido interpopulacional foi tomado como variável dependente (y) e os ciclos de seleção como variável independente $(\mathrm{x})$, o coeficiente de regressão linear $\left(b_{1}\right)$ forneceu a estimativa do progresso genético por ciclo e, com a divisão do $b_{1}$ pelo intercepto $\left(b_{0}\right)$, foi obtido o progresso genético em percentagem; ii, pela diferença entre as médias dos híbridos interpopulacionais $\mathrm{C} 2$ e $\mathrm{C} 0$ dividida por dois (número de ciclos seletivos completados), que permitiu estimar o progresso genético, tendo-se considerado os dados de todas as avaliações. Para obter o progresso genético em percentagem, o seu valor foi dividido pela média do híbrido $\mathrm{C} 0$.

\section{Resultados e Discussão}

A análise de variância, que considerou os dados da safra de 2005/2006, não detectou diferença entre os tratamentos em nenhuma das características (Tabela 1). Contudo, na safra seguinte, observou-se diferença entre os híbridos interpopulacionais do $\mathrm{C} 0$ e C2 somente para PE. Isso demonstra que, quando as diferenças entre os tratamentos não são de grande magnitude, é necessário utilizar maior número de

Tabela 1. Resumo da análise de variância individual para produtividade de espigas despalhadas (PE) e prolificidade (PLF) na avaliação do progresso genético com a seleção recorrente recíproca nas safras 2005/2006 e 2006/2007.

\begin{tabular}{|c|c|c|c|c|}
\hline \multirow{2}{*}{$\begin{array}{l}\text { Fonte de } \\
\text { variação }\end{array}$} & \multicolumn{2}{|c|}{ Safra $2005 / 2006$} & \multicolumn{2}{|c|}{ Safra 2006/2007 } \\
\hline & QM & $\operatorname{Pr}>F$ & QM & $\operatorname{Pr}>F$ \\
\hline & \multicolumn{4}{|c|}{ Produtividade de espigas despalhadas } \\
\hline Local (L) & 353,3367 & 0,00 & 0,9139 & 0,55 \\
\hline Híbridos (H) & 2,2063 & 0,79 & 130,5862 & 0,00 \\
\hline $\mathrm{LxH}$ & 4,9610 & 0,39 & 0,4595 & 0,67 \\
\hline Erro & 4,6538 & - & 2,5005 & - \\
\hline \multirow[t]{2}{*}{$\mathrm{CV}(\%)$} & 22,77 & - & 15,96 & - \\
\hline & \multicolumn{4}{|c|}{ Prolificidade } \\
\hline Local (L) & 0,0342 & 0,04 & 0,0016 & 0,84 \\
\hline Híbridos $(\mathrm{H})$ & 0,0072 & 0,44 & 1,1799 & 0,00 \\
\hline LxH & 0,0064 & 0,50 & 0,0254 & 0,43 \\
\hline Erro & 0,0071 & - & 0,0411 & - \\
\hline CV (\%) & 8,97 & - & 19,01 & - \\
\hline
\end{tabular}


repetições para discriminá-los. Em virtude de a soma de quadrados para a interação entre tratamentos e locais praticamente não ter contribuído com a soma de quadrados totais, as análises conjuntas foram efetuadas tendo-se considerado o somatório das repetições dos dois locais.

As análises de variância conjuntas das safras 2005/2006 e 2006/2007, onde somente os tratamentos $\mathrm{C}_{1} \times \mathrm{CO}_{2}$ e $\mathrm{C}_{1} \times \mathrm{C}_{2}$ foram incluídos, mostrou diferenças significativas em $\operatorname{PE}(p<0,01)$ e $\operatorname{PLF}(p<0,05)$ (Tabela 2). Não houve diferenças na interação entre tratamentos e safras, o que indica que o comportamento dos híbridos interpopulacionais $\mathrm{C}_{1} \times \mathrm{CO}_{2}$ e $\mathrm{C}_{1} \times \mathrm{C}_{2}$ foi coincidente nas duas safras.

A produtividade de espigas despalhadas do $\mathrm{C}_{1} \times \mathrm{CO}_{2}$ foi semelhante à do $\mathrm{HDF}_{1}$ e não diferiu significativamente (Tabela 3). Portanto, o desempenho dos híbridos obtidos a partir das gerações $\mathrm{F}_{1}\left(\mathrm{HDF}_{1}\right)$ ou $\mathrm{F}_{2}\left(\mathrm{CO}_{1} \times \mathrm{CO}_{2}\right)$ de dois híbridos simples são similares, o que corrobora os relatos de Souza Sobrinho et al. (2002).

Tabela 2. Resumo da análise de variância conjunta das safras 2005/2006 e 2006/2007 para produtividade de espigas despalhadas (PE) e prolificidade (PLF) na avaliação do progresso genético com a seleção recorrente recíproca.

\begin{tabular}{lcccc}
\hline Fonte de variação & QM & Pr $>$ F & QM & $\operatorname{Pr}>$ F \\
\hline & $----------P E---------$ & $---------P L F---------$ \\
Ano (A) & 23,3633 & 0,00 & 0,2383 & 0,00 \\
Híbridos (H) & 32,4993 & 0,00 & 0,1011 & 0,04 \\
AxH & 3,8672 & 0,21 & 0,0671 & 0,10 \\
Erro & 2,4643 & - & 0,0237 & - \\
\hline CV $(\%)$ & 16,06 & - & 14,34 & -
\end{tabular}

Tabela 3. Produtividade de espigas despalhadas (PE) e prolificidade (PLF) de híbridos interpopulacionais, híbridos simples parentais (HS) e híbridos duplos entre eles (HD), na avaliação da seleção recorrente recíproca em milho na safra de $2005 / 2006^{(1)}$.

\begin{tabular}{lcc}
\hline Tratamentos & $\begin{array}{c}\text { PE } \\
\left(\mathrm{Mg} \mathrm{ha}^{-1}\right)\end{array}$ & $\begin{array}{c}\text { PLF } \\
\text { (espigas por planta) }\end{array}$ \\
\hline $\mathrm{C}_{1} \times \mathrm{CO}_{2}$ & $8,91 \mathrm{a}$ & $0,961 \mathrm{a}$ \\
$\mathrm{C}_{1} \times \mathrm{Cl}_{2}$ & $9,31 \mathrm{a}$ & $0,966 \mathrm{a}$ \\
$\mathrm{C}_{2} \times \mathrm{C}_{2}$ & $9,84 \mathrm{a}$ & $0,975 \mathrm{a}$ \\
$\mathrm{HD} \mathrm{F}_{1}$ & $9,05 \mathrm{a}$ & $0,906 \mathrm{a}$ \\
$\mathrm{HS} 1$ & $10,13 \mathrm{a}$ & $0,983 \mathrm{a}$ \\
$\mathrm{HS} 2$ & $9,58 \mathrm{a}$ & $0,892 \mathrm{a}$ \\
\hline
\end{tabular}

${ }^{(1)}$ Médias seguidas por letras iguais na coluna não diferem entre si pelo teste de Tukey, a $5 \%$ de probabilidade.

Pesq. agropec. bras., Brasília, v.44, n.12, p.1667-1672, dez. 2009
Houve tendência de incremento tanto na PE quanto na PLF, confirmada pela estimativa do coeficiente de regressão linear (Figura 1 e 2). A estimativa de $b_{1}$ $(0,465)$ para PE foi diferente de zero, o que permite inferir que a SRR proporcionou ganho genético de $465 \mathrm{~kg} \mathrm{ha}^{-1}$ por ciclo. Tendo como referência o híbrido interpopulacional do $\mathrm{C} 0$, representado pela estimativa de $\mathrm{b}_{0}$, esse valor corresponde a um progresso genético de $5,2 \%$ por ciclo. Quanto à PLF, a estimativa do progresso genético de $0,7 \%$ por ciclo, obtida por meio da equação de regressão linear, não diferiu de zero (Figura 2).

Para confirmar o progresso genético com a SRR na safra de 2005/2006, os híbridos interpopulacionais $\mathrm{C}_{1} \times \mathrm{CO}_{2}$ e $\mathrm{C}_{1} \times \mathrm{C}_{2}$ foram novamente avaliados na safra de 2006/2007. Nas duas safras avaliadas, as médias de $\mathrm{C} 2{ }_{1} \times \mathrm{C}_{2}$ foram superiores às de $\mathrm{CO}_{1} \times \mathrm{CO}_{2}$ em $15,74 \%$ (10,0 e $8,64 \mathrm{Mg} \mathrm{ha}^{-1}$, respectivamente) para PE e em 7\% para PLF (1,06 e 0,99 espigas por planta, respectivamente), o que confirma a eficiência da SRR. Os progressos genéticos estimados foram superiores aos da safra de 2005/2006 e, nesse caso, as estimativas foram obtidas a partir de um maior número de informações.

Com a média das estimativas do progresso genético por ciclo $(7,9 \%)$ obtidas neste estudo para PE,

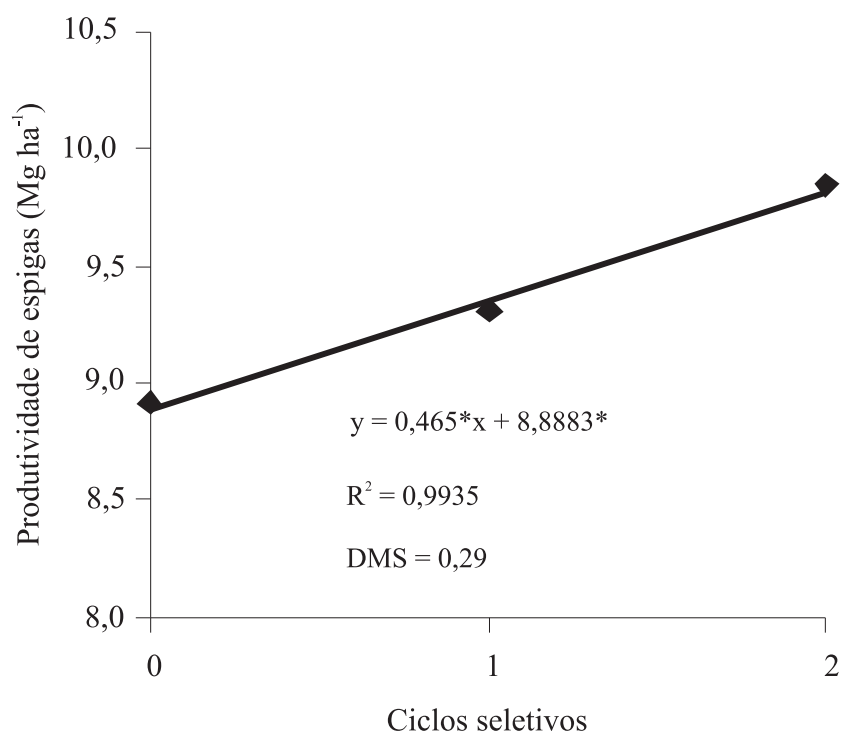

Figura 1. Produtividade de espigas despalhadas de híbridos interpopulacionais de milho com a seleção recorrente recíproca. *Diferente de zero pelo teste $\mathrm{t}$, a $5 \%$ de probabilidade; DMS, diferença mínima significativa, a 5\% de probabilidade. 
observa-se coerência com os relatos de SRR presentes na literatura. Trabalhos com populações derivadas de híbridos simples mostraram respostas de 5,7 (Raposo et al., 2004), 4,5 e 6,2\% (Coors, 1999) à seleção por ciclo. Em programas com populações temperadas (BS10 e BS11) submetidas à SRR de irmãos germanos por oito ciclos, Eyherabide \& Hallauer (1991) reportaram ganho de $6,5 \%$ por ciclo. Keeratinijakal \& Lamkey (1993), ao trabalhar com as populações BSSS e BSBC1 por 11 ciclos, relataram ganho de $6,95 \%$ com a seleção por ciclo. Santos et al. (2007) obtiveram resposta média de $7,2 \%$ à seleção após três ciclos com as populações de milho tropical EPB4 e EPB5.

Os resultados do progresso genético apresentados para PLF foram inferiores aos de Souza Júnior \& Pinto (2000), que observaram resposta de 11,8\% à seleção por ciclo. Contudo, a média no híbrido interpopulacional no $\mathrm{C} 2$ desse estudo foi de 1,06 espiga por planta, muito próxima à média de 1,10 apresentada pelo híbrido interpopulacional do C3 no estudo de Souza Júnior \& Pinto (2000). Como essa característica não foi considerada na seleção, o progresso genético observado pode ser atribuído à seleção massal praticada no momento da obtenção das progênies. Esse fato também foi observado por Souza Júnior \& Pinto (2000) e reforçado por relatos sobre a eficiência da

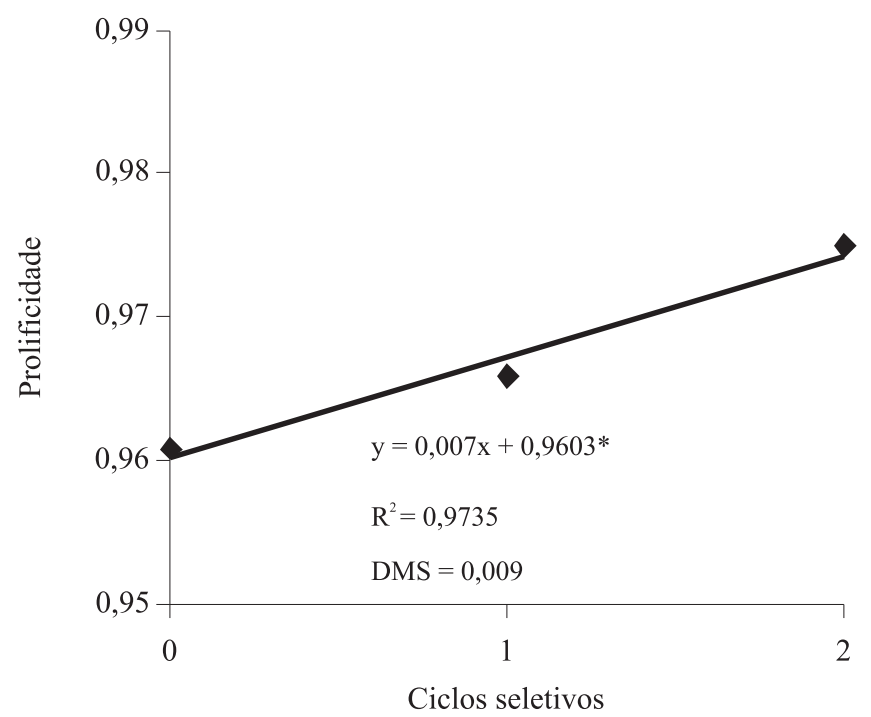

Figura 2. Prolificidade (espigas por planta) de híbridos interpopulacionais de milho com a seleção recorrente recíproca. *Diferente de zero pelo teste $\mathrm{t}$ a $1 \%$ de probabilidade; DMS, diferença mínima significativa, a 5\% de probabilidade. seleção massal para essa característica (Maita \& Coors, 1996; Bento et al., 2003). Além disso, a correlação genética positiva entre PE e PLF pode ser associada ao ganho genético observado para PLF, o que também foi relatado por Câmara et al. (2007).

A alternância entre progênies de $S_{1}$ e de meios-irmãos como unidades de recombinação mostrou-se vantajosa, permitindo realizar simultaneamente a recombinação e a obtenção das progênies para o ciclo subsequente. Com isso, cada ciclo pode ser concluído em um ano, o que supera um dos principais entraves da SRR, que é a longa duração dos ciclos. Como consequência, o progresso genético por ciclo por ano foi pelo menos o dobro dos relatados na literatura, nos quais cada ciclo tinha a duração mínima de dois anos.

$\mathrm{O}$ híbrido interpopulacional $\mathrm{C} 2_{1} \times \mathrm{C}_{2}$ apresentou produtividade média muito semelhante à dos dois híbridos simples parentais, de reconhecido potencial produtivo (Tabela 3). Infere-se que a SRR possibilita obter híbridos interpopulacionais com desempenho comparável aos melhores híbridos simples existentes no mercado. Os híbridos interpopulacionais assim obtidos podem ser utilizados pelos agricultores familiares, uma vez que a produção de sementes é facilitada nesse tipo de híbrido, pois os parentais são duas populações em equilíbrio de Hardy-Weinberg, com boa produtividade de grãos.

\section{Conclusão}

A seleção recorrente recíproca é eficiente para promover o progresso genético em $7,9 \%$ por ciclo para produtividade de espigas despalhadas nos híbridos interpopulacionais obtidos a partir de populações $\mathrm{F}_{2}$ de híbridos simples de milho.

\section{Agradecimentos}

À Fundação de Amparo à Pesquisa do Estado de Minas Gerais, pelo financiamento do projeto, e ao Conselho Nacional de Desenvolvimento Científico e Tecnológico, pela bolsa concedida ao primeiro autor.

\section{Referências}

BENTO, D.A.V.; RAMALHO, M.A.P.; SOUZA, J.C. de. Seleção massal para prolificidade em milho na época normal e na "safrinha". Revista Brasileira de Milho e Sorgo, v.2, p.78-87, 2003.

CÂMARA, T.M.M.; BENTO, D.A.V.; ALVES, G.F.; SANTOS, M.F.; MOREIRA, J.U.V.; SOUZA JÚNIOR, C.L. Parâmetros 
genéticos de caracteres relacionados à tolerância à deficiência hídrica em milho tropical. Bragantia, v.66, p.595-603, 2007.

COMSTOCK, R.E.; ROBINSON, H.F.; HARVEY, P.H. A breeding procedure designed to make maximum use of both general and specific combining ability. Agronomy Journal, v.41, p.360-367, 1949.

COORS, J.G. Selection methodologies and heterosis. In: COORS, J.G.; PANDEY, S. (Ed.). Genetics and exploitation of heterosis in crops. Madison: American Society of Agronomy, 1999. 524p.

EYHERABIDE, G.H.; HALLAUER, A.R. Reciprocal full-sib recurrent selection in maize: I direct and indirect responses. Crop Science, v.31, p.952-959, 1991.

KEERATINIJAKAL, V.; LAMKEY, K.R. Responses to reciprocal recurrent selection in BSS and BCSB1 maize populations. Crop Science, v.33, p.73-73, 1993.

MAITA, R.; COORS, J.G. Twenty cycles of biparental mass selection for prolificacy in the open-pollinated maize population Golden Glow. Crop Science, v.36, p.1527-1532, 1996.

PATERNIANI, E.; VENCOVSKY, R. Reciprocal recurrent selection based on half-sib progenies and prolific plants in maize (Zea mays L.). Maydica, v.23, p.209-219, 1978.

PATERNIANI, E.; VENCOVSKY, R. Reciprocal recurrent selection in maize (Zea mays L.) based on testcross of half-sib families. Maydica, v.22, p.141-152, 1977.
RAPOSO, F.V.; RAMALHO, M.A.P.; RIBEIRO, P.H.E. Alterations in heterosis of maize populations derived from single-cross hybrids after a reciprocal recurrent selection. Crop Breeding and Applied Biotechnology, v.4, p.74-80, 2004.

SANTOS, M.F.; CÂMARA, T.M.M.; MORO, G.V.; COSTA, E.F.N.; SOUZA JÚNIOR, C.L. de. Responses to selection and changes in combining ability after three cycles of a modified reciprocal recurrent selection in maize. Euphytica, v.157, p.185-194, 2007.

SAS INSTITUTE. SAS: user's guide statistical. Version 8. Cary: SAS Institute, 2000.

SOUZA JÚNIOR, C.L. de. Reciprocal recurrent selection with half-sib progenies obtained alternately from non-inbred $\left(\mathrm{S}_{0}\right)$ and inbred $\left(\mathrm{S}_{1}\right)$ plants in maize. Maydica, v.22, p.19-31, 1987.

SOUZA JÚNIOR, C.L. de; PINTO, R.M.C. Responses to a short-term reciprocal recurrent selection procedure in maize. Maydica, v.45, p.21-28, 2000.

SOUZA SOBRINHO, F. de; RAMALHO, M.A.P.; SOUZA, J.C. de. Alternatives for obtaining double cross maize hybrids. Revista Brasileira de Milho e Sorgo, v.1, p.70-76, 2002.

WRICKE, G.; WEBER, W.E. Quantitative genetics and selection in plant breeding. New York: Walter de Gruyter, 1986. 406p.

Recebido em 28 de setembro de 2009 e aprovado em 19 de novembro de 2009 